\title{
A Dramatic Improvement by Ventriculoperitoneal Shunt Surgery in a Patient With Secondary Normal Pressure Hydrocephalus
}

\author{
Halil Onder ${ }^{\mathrm{a}, \mathrm{c}}$, Umut D. Akturk ${ }^{\mathrm{b}}$
}

A 60-year-old male patient was admitted to our clinic with symptoms of gait difficulty, urinary incontinence, and cognitive impairment which had started over the last 5 years and gradually progressed. So he had to use unilateral support for outdoor activities over the last 1-year period. Besides, he had severe symptoms of urinary incontinence and had to use underpads for a long time. Upon interrogation of medical history, it was learned that he had been hospitalized in the intensive care unit for 1 month due to post-traumatic subarachnoid hemorrhage (SAH) and right frontal hemorrhage which had occurred due to motor vehicle accident 40 years ago. At admission to our clinic, he was orientated and cooperative. However, it was noticed that the patient was uninterested to the interview and slowing in the reaction time was apparent. He was not willing to state his symptoms and rather did not speak spontaneously which was compatible with apathetic state. Neurological examinations of the pyramidal, sensory and cerebellar functions were within normal limits. However, gait was evaluated as wide-based, short-stepped, slow and shuffling. He had difficulty particularly during turning, and episodes of freezing of gait were apparent (Video images are unavailable due to technical problems). Standardized mini-mental test (SMMT) score was evaluated as 19 points. Cranial magnetic resonance imaging (MRI) showed left frontal encephalomalacia and non-obstructive hydrocephalus (Fig. 1). Taken together, normal pressure hydrocephalus was considered at the forefront, and cerebrospinal fluid (CSF) tap test (TT) was suggested. CSF opening pressure was $10 \mathrm{~cm}$ $\mathrm{H}_{2} \mathrm{O}$, and $40 \mathrm{~mL}$ CSF was drained which provided a temporary, significant improvement in the patient's locomotion and symptoms of urinary incontinence. Taken together, the diagnosis of secondary normal pressure hydrocephalus (sNPH) was established. The patient and his relatives were informed about the disease, and ventriculoperitoneal shunt (VP) surgery was suggested. After taking informed consent, surgery was performed. Following VP shunt, a significant recovery in the gait and cognitive symptoms were achieved. Cranial computed tomography

Manuscript submitted June 26, 2019, accepted August 5, 2019

${ }^{a}$ Neurology Clinic, Yozgat City Hospital, Yozgat, Turkey

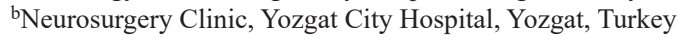

${ }^{\mathrm{c}}$ Corresponding Author: Halil Onder, Neurology Clinic, Yozgat City Hospital, Yozgat, Turkey. Email: halilnder@yahoo.com

doi: https://doi.org/10.14740/jnr545 performed 1 week after shunt surgery, showed an intact shunt (Fig. 2). On the second month of follow-up after surgery, he had no longer need for support, and all the parameters of his gait improved dramatically (Supplementary video 1, www. neurores.org). Urinary continence symptoms were nearly over. His perception and communication, as well as interest had improved significantly and he got 24 points on SMMT.

Approximately, $50 \%$ of the normal pressure hydrocephalus (NPH) cases occur in association with prior events that cause the development of this syndrome [1]. In the recent crucial report by Daou et al, it was concluded that sNPH should be differentiated from idiopathic NPH (iNPH) based on outcome and on clinical, pathophysiological, and epidemiological characteristics, but should not be considered as a separate entity [2]. The better response to shunt surgery in sNPH (in comparison to iNPH) has also been emphasized in other studies [3]. The diagnosis of sNPH is based on a combination of clinical history, physical examination, and imaging studies [2]. In our patient, the clinical presentation was characterized by the classic triad of dementia, gait disturbance, and urinary incontinence. Besides, cranial MRI showed hydrocephalus and CSF TT provided a significant improvement (and CSF opening pressure was normal). The remarkable point was that he had a history of traumatic SAH and parenchymal hemorrhage 40 years ago, but clinical symptoms were rather recognized over the last 5 years. Considering the long interval between the event of trauma and clinical onset of symptoms, we think that it may be challenging to conclude a clear causal association between these two occurrences. It has been indicated that for diagnosis of sNPH, there should be a well-established event to induce NPH and the clinical manifestations generally should begin immediately or several months after the event. However, the time required for the development of NPH after the inducer event (SAH, trauma, meningitis, stroke, tumors, etc.) has not been established properly. Combining the clinical manifestations, medical history of a traumatic brain injury and clear evidence in neuroimaging, we have established the final diagnosis of sNPH. In accordance with the literature data, a dramatic improvement was obtained following VP shunt surgery, such that his gait problems were determined to be totally resolved on the second month of follow-up. At this point, we would like to state that in our province with a population of approximately 500,000 , our clinic is a senior center where the substantial rate of the patients with suspicion of NPH is eval- 


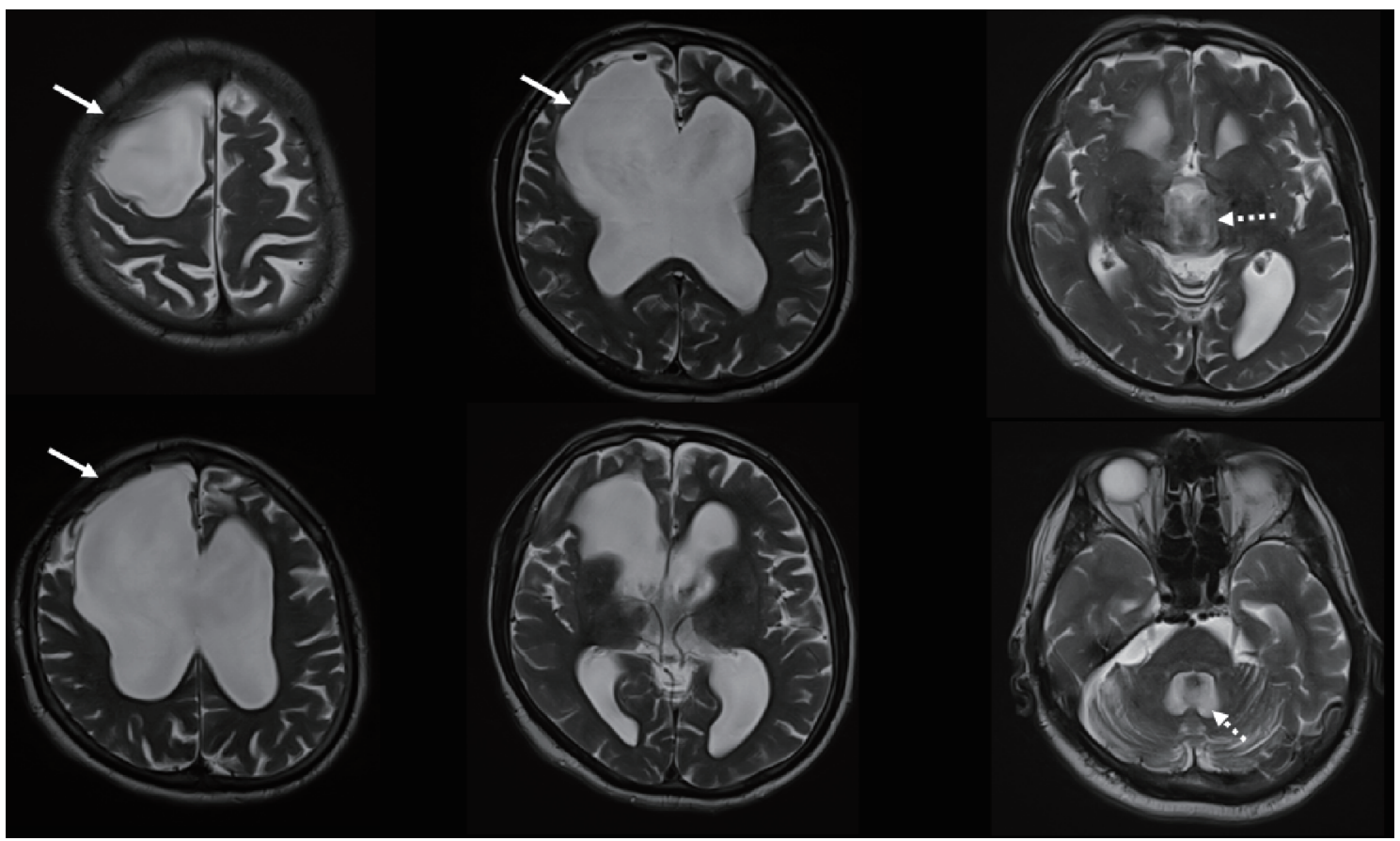

Figure 1. Cranial MRI shows obstructive hydrocephalus and righ frontal encephalomalacia (jagged arrows shows enlargement of third and fourth ventricles). MRI: magnetic resonance imaging.

uated. Although we have not performed a systematical analysis, our observation is that most of the NPH patients in our cohort were diagnosed with iNPH and we generally observe a mild or moderate post-shunt response in patients with iNPH, whereas it is substantially more significant in subgroup of patients with sNPH. In the light of these data, via the illustration of this remarkable post-shunt response in our case and related literature data, we would like to draw attention to the

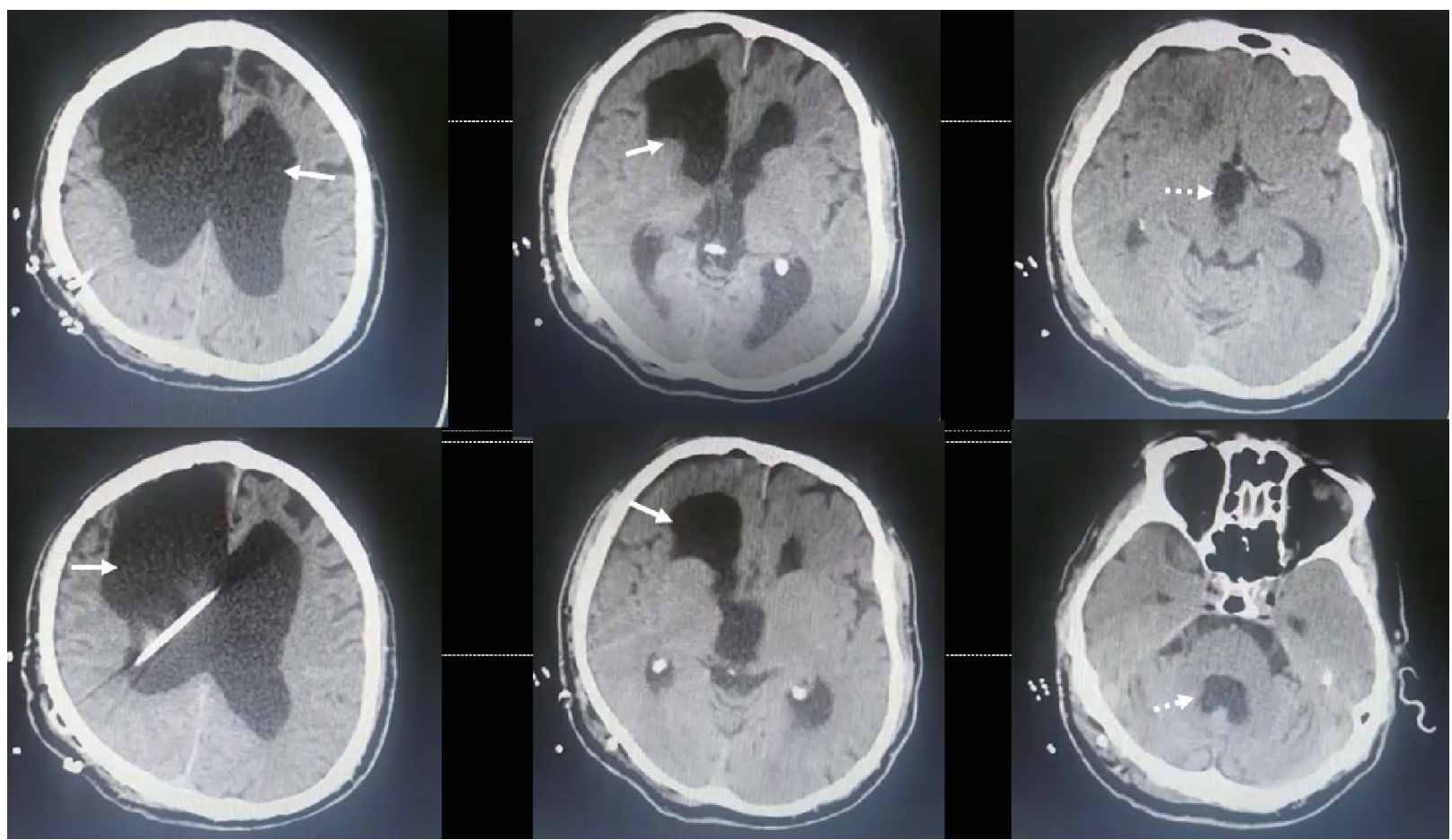

Figure 2. Cranial computed tomography, recorded 1 week after VP shunt surgery, shows an intact shunt. VP: ventriculoperitoneal shunt. 
issue of better clinical outcomes in patients with sNPH. Although this is a known entity in literature, we think that our case may constitute a crucial illustration in this regard and remark the need for further investigations. Future reports focusing on this differing treatment response and probably differing pathophysiology in the subgroup of NPH patients with secondary etiology may provide substantial contributions regarding the unknown aspects of the physiology of CSF, as well as the pathophysiology of various CSF flow disorders.

\section{Acknowledgments}

None to declare.

\section{Financial Disclosure}

None to declare.

\section{Conflict of Interest}

None to declare.

\section{Informed Consent}

Informed consent has been obtained from the patient.

\section{Author Contributions}

HO contributed substantially to clinical evaluation, conception and design of the report, writing, critical review and submission of the manuscript. UDA contributed substantially to clinical evaluation and critical review of the manuscript.

\section{References}

1. Bradley WG. Normal pressure hydrocephalus: new concepts on etiology and diagnosis. AJNR Am J Neuroradiol. 2000;21(9): 1586-1590.

2. Daou B, Klinge P, Tjoumakaris S, Rosenwasser RH, Jabbour P. Revisiting secondary normal pressure hydrocephalus: does it exist? A review. Neurosurg Focus. 2016;41(3):E6.

3. Vanneste JA. Diagnosis and management of normal-pressure hydrocephalus. J Neurol. 2000;247(1):5-14. 